\title{
ON REFINEMENTS OF HADAMARD'S INEQUALITIES
}

\author{
GOU-SHENG YANG AND CHUNG-SHIN WANG
}

\begin{abstract}
Some refinements of Hadamard's inequalities are established.
\end{abstract}
\section{Introduction}

The inequalities

$$
f\left(\frac{a+b}{2}\right) \leq \frac{1}{b-a} \int_{a}^{b} f(x) d x \leq \frac{f(a)+f(b)}{2}
$$

which holds for all convex functions $f:[a, b] \rightarrow R$ are known in the literature as Hadamard's inequalities. In [2] and [3], S. S. Dragomir established some refinements of the first inequality of (1.1). In [4], G. S. Yang and M. C. Hong established a refinement of the second inequality of (1.1).

The main purpose of this note is to establish further generalization of the results in [2], [3] and [4].

As in [1] and [2], let $E$ be a nonempty set and let $L$ be a linear class of real-valued functions from $E$ to $R$ having the properties:

$$
\begin{aligned}
& L_{1}: f, g \in L \Rightarrow(a f+b g) \in L \text { for all } a, b \in R \\
& L_{2}: 1 \in L, \text { that is, if } f(t)=1(t \in E), \text { then } f \in L .
\end{aligned}
$$

A linear functional $A: L \rightarrow R$ is isotonic if

$$
\begin{aligned}
& A_{1}: A(a f+b g)=a A(f)+b A(g) \text { for } f, g \in L \text { and } a, b \in R \\
& A_{2}: f \in L, \quad f(t) \geq 0 \text { on } E \Rightarrow A(f) \geq 0 \quad(A \text { is isotonic }) .
\end{aligned}
$$

We need the following Jensen's inequality (see [1] or [2]).

Jemsen's inequality. Let $L$ satisfy the above properties on $E$, and suppose $\Phi$ is a convex function on an interval $I \subseteq R$. If $A$ is any isotonic linear functional with $A(1)=1$, then, for all $g \in L$ such that $\Phi(g) \in L$, we have $A(g) \in I$ and $\Phi(A(g)) \leq A(\Phi(g))$.

Received April 19, 1996.

1991 Mathematics Subject Classification. Primary 26D15.

Key words and phrases. Hadamard's inequality, Jensen's inequality, convex function, isotonic linear functional. 


\section{Preliminary Lemmas}

In order to establish the main theorems, we start with the following lemmas.

Lemma 1. Let $C$ be a convex subset of a real linear space $X$, and $f: C \rightarrow R$, the real numbers, be a convex function. Let $a_{i}>0(i=1,2, \ldots, n)$ with $\sum_{i=1}^{n} a_{i}=1$ and $a=\min _{1 \leq i \leq n}\left\{a_{i}\right\}$. Given a sequence $x=\left\{x_{i}, x_{2}, \ldots, x_{n}\right\}$ in $C$, let $\Phi_{x}:\left[0, n_{a}\right] \rightarrow R$ be defined by

$$
\Phi_{x}(t)=\sum_{i=1}^{n} a_{i} f\left(\left[1-\frac{g(t)}{n a_{i}}\right] x_{i}+\frac{g(t)}{n a_{i}} x_{i+1}\right),
$$

where $g$ is a linear function on $[0, n a]$ such that $0 \leq g(t) \leq n a$ and $x_{n+1}=x_{1}$. Then

(1) $\Phi_{x}$ is convex on $[0, n a]$,

(2) $f\left(\sum_{i=1}^{n} a_{i} x_{i}\right) \leq \Phi_{x}(t) \leq \sum_{i=1}^{n} a_{i} f\left(x_{i}\right)$ for all $t \in[0, n a]$.

Proof. Let $t_{1}, t_{2} \in[0, n a]$ and $\alpha, \beta \geq 0$ with $\alpha+\beta=1$. Since $f$ is convex on $C$ and $g$ is linear in $[0, n a]$, we have

$$
\begin{aligned}
& \Phi_{x}\left(\alpha t_{1}+\beta t_{2}\right)= \sum_{i=1}^{n} a_{i} f\left(\left[1-\frac{g\left(\alpha t_{1}+\beta t_{2}\right)}{n a_{i}}\right] x_{i}+\frac{g\left(\alpha t_{1}+\beta t_{2}\right)}{n a_{i}} x_{i+1}\right) \\
&= \sum_{i=1}^{n} a_{i} f\left(\alpha\left[\left(1-\frac{g\left(t_{1}\right)}{n a_{i}}\right) x_{i}+\frac{g\left(t_{1}\right)}{n a_{i}} x_{i+1}\right]\right. \\
&\left.+\beta\left[\left(1-\frac{g\left(t_{2}\right)}{n a_{i}}\right) x_{i}+\frac{g\left(t_{2}\right)}{n a_{i}} x_{i+1}\right]\right) \\
& \leq \alpha \sum_{i=1}^{n} a_{i} f\left(\left[1-\frac{g\left(t_{1}\right)}{n a_{i}}\right] x_{i}+\frac{g\left(t_{1}\right)}{n a_{i}} x_{i+1}\right) \\
& \quad+\beta \sum_{i=1}^{n} a_{i} f\left(\left[1-\frac{g\left(t_{2}\right)}{n a_{i}}\right] x_{i}+\frac{g\left(t_{2}\right)}{n a_{i}} x_{i+1}\right) \\
&=\alpha \Phi_{x}\left(t_{1}\right)+\beta \Phi_{x}\left(t_{2}\right) .
\end{aligned}
$$

This completes the proof of (1).

Next, using the convexity of $f$ and note that $x_{n+1}=x_{1}$, we have

$$
\begin{aligned}
\Phi_{x}(t) & \leq \sum_{i=1}^{n} a_{i}\left[\left(1-\frac{g(t)}{n a_{i}}\right) f\left(x_{i}\right)+\frac{g(t)}{n a_{i}} f\left(x_{i+1}\right)\right] \\
& =\sum_{i=1}^{n} a_{i} f\left(x_{i}\right)+\frac{g(t)}{n} \sum_{i=1}^{n}\left[f\left(x_{i+1}\right)-f\left(x_{i}\right)\right]=\sum_{i=1}^{n} a_{i} f\left(x_{i}\right)
\end{aligned}
$$


and

$$
\begin{aligned}
\Phi_{x}(t) & \geq f\left(\sum_{i=1}^{n} a_{i}\left[\left(1-\frac{g(t)}{n a_{i}}\right) x_{i}+\frac{g(t)}{n a_{i}} x_{i+1}\right]\right) \\
& =f\left(\sum_{i=1}^{n} a_{i} x_{i}+\frac{g(t)}{n} \sum_{i=1}^{n}\left[x_{i+1}-x_{i}\right]\right)=f\left(\sum_{i=1}^{n} a_{i} x_{i}\right)
\end{aligned}
$$

for all $t \in[0, n a]$. This proves (2).

Remark $\mathbb{1}$. Lemma 2.1 in [2] is the special case of our lemma 1 when $n=2, g(t)=t$ and $a_{1}=a_{2}=\frac{1}{2}$.

In [4], G. S. Yang and M. C. Hong proved:

Lemma 2. If $f:[a, b] \rightarrow R$ is a convex function and $F:[0,1] \rightarrow R$ is defined by

$$
F(t)=\frac{1}{2(b-a)} \int_{a}^{b}\left\{f\left(\left[\frac{1+t}{2}\right] a+\left[\frac{1-t}{2}\right] x\right)+f\left(\left[\frac{1+t}{2}\right] b+\left[\frac{1-t}{2}\right] x\right)\right\} d x
$$

then $F$ is convex, increasing on $[0,1]$ and

$$
\frac{1}{b-a} \int_{a}^{b} f(x) d x=F(0) \leq F(t) \leq F(1)=\frac{f(a)+f(b)}{2} .
$$

They used the differentiability of $f$ on $(0,1)$ to prove $F$ is increasing on $[0,1]$. Here, we give a proof without using the differentiability of $f$ on $(0,1)$ as follows:

Proof. That $F$ is convex on $[0,1]$ is easy to verify. Now, if $0 \leq t<1$, then

$$
\begin{aligned}
F(t) & =\frac{1}{2(b-a)} \int_{a}^{b}\left\{f\left(\frac{[1+t] a+[1-t] x}{2}\right)+f\left(\frac{[1+t] b+[1-t] x}{2}\right)\right\} d x \\
& =\frac{1}{(1-t)(b-a)}\left\{\int_{a}^{\frac{a+b}{2}-t\left(\frac{b-a}{2}\right)} f(x) d x+\int_{\frac{a+b}{2}+t\left(\frac{b-a}{2}\right)}^{b} f(x) d x\right\} .
\end{aligned}
$$

Since $f$ is convex, we have

$$
\begin{aligned}
F^{\prime}(t)= & \frac{1}{(1-t)^{2}(b-a)}\left\{\int_{a}^{\frac{a+b}{2}-t\left(\frac{b-a}{2}\right)} f(x) d x+\int_{\frac{a+b}{2}+t\left(\frac{b-a}{2}\right)}^{b} f(x) d x\right\} \\
& +\frac{1}{(1-t)(b-a)}\left\{f\left(\frac{a+b}{2}-t\left[\frac{b-a}{2}\right]\right)\left[-\frac{b-a}{2}\right]\right. \\
& \left.-f\left(\frac{a+b}{2}+t\left[\frac{b-a}{2}\right]\right)\left[\frac{b-a}{2}\right]\right\} \\
= & \frac{1}{(1-t)^{2}(b-a)}\left\{\int_{a}^{\frac{a+b}{2}-t\left(\frac{b-a}{2}\right)} f(x) d x+\int_{\frac{a+b}{2}+t\left(\frac{b-a}{2}\right)}^{b} f(x) d x\right\}
\end{aligned}
$$




$$
\begin{aligned}
& -\frac{1}{2(1-t)}\left\{f\left(\frac{a+b}{2}-t\left[\frac{b-a}{2}\right]\right)+f\left(\frac{a+b}{2}+t\left[\frac{b-a}{2}\right]\right)\right\} \\
\geq & \frac{1}{2(1-t)}\left\{f\left(\left[\frac{3+t}{4}\right] a+\left[\frac{1-t}{4}\right] b\right)+f\left(\left[\frac{1-t}{4}\right] a+\left[\frac{3+t}{4}\right] b\right)\right\} \\
& -\frac{1}{2(1-t)}\left\{f\left(\left[\frac{1+t}{2}\right] a+\left[\frac{1-t}{2}\right] b\right)+f\left(\left[\frac{1-t}{2}\right] a+\left[\frac{1+t}{2}\right] b\right)\right\} \\
= & \frac{1}{2(1-t)}\left\{f\left(\left[\frac{1-t}{4}\right] a+\left[\frac{3+t}{4}\right] b\right)-f\left(\left[\frac{1-t}{2}\right] a+\left[\frac{1+t}{2}\right] b\right)\right\} \\
& \quad-\frac{1}{2(1-t)}\left\{f\left(\left[\frac{1+t}{2}\right] a+\left[\frac{1-t}{2}\right] b\right)-f\left(\left[\frac{3+t}{4}\right] a+\left[\frac{1-t}{4}\right] b\right)\right\} \\
\geq 0 . &
\end{aligned}
$$

This shows that $F$ is increasing on $[0,1]$. Hence

$$
\frac{1}{b-a} \int_{a}^{b} f(x) d x=F(0) \leq F(t) \leq F(1)=\frac{f(a)+f(b)}{2} .
$$

This completes the proof.

\section{Main Results}

Now, we give our main results as the following theorems.

Theorem $\mathbb{1}$. Under the conditions of Lemma 1, let $L$, A satisfy the conditions $L_{1}$, $L_{2}, A_{1}$ and $A_{2}$, and let $h: E \rightarrow[0, n a]$ be a function such that $h \in L$ and

$$
f\left(\left[1-\frac{g(h)}{n a_{i}}\right] x_{i}+\frac{g(h)}{n a_{i}} x_{i+1}\right) \in L \quad \text { for } i=1,2, \ldots, n .
$$

If $A(1)=1$, then

$$
f\left(\sum_{i=1}^{n} a_{i} x_{i}\right) \leq \Phi_{x}(A(h)) \leq A\left(\Phi_{x}(h)\right) \leq \sum_{i=1}^{n} a_{i} f\left(x_{i}\right) .
$$

Proof. Using Jensen's inequality, we have

$$
\Phi_{x}(A(h)) \leq A\left(\Phi_{x}(h)\right) .
$$

This is the second inequality in (3.1).

Since $f$ is convex on $C$ and $A$ is an istonic linear functional on $L$, we have

$$
\begin{aligned}
\Phi_{x}(A(h)) & =\sum_{i=1}^{n} a_{i} f\left(\left[1-\frac{g(A(h))}{n a_{i}}\right] x_{i}+\frac{g(A(h))}{n a_{i}} x_{i+1}\right) \\
& \geq f\left(\sum_{i=1}^{n} a_{i}\left[\left(1-\frac{g(A(h))}{n a_{i}}\right) x_{i}+\frac{g(A(h))}{n a_{i}} x_{i+1}\right]\right)=f\left(\sum_{i=1}^{n} a_{i} x_{i}\right) .
\end{aligned}
$$


This is the first inequality of (3.1).

Finally,

$$
\begin{aligned}
\Phi_{x}(h) & =\sum_{i=1}^{n} a_{i} f\left(\left[1-\frac{g(h)}{n a_{i}}\right] x_{i}+\frac{g(h)}{n a_{i}} x_{i+1}\right) \\
& \leq \sum_{i=1}^{n} a_{i}\left[\left(1-\frac{g(h)}{n a_{i}}\right) f\left(x_{i}\right)+\frac{g(h)}{n a_{i}} f\left(x_{i+1}\right)\right]=\sum_{i=1}^{n} a_{i} f\left(x_{i}\right) .
\end{aligned}
$$

Using $A_{1}, A_{2}$ and $A(1)=1$, we have $A\left(\Phi_{x}(h)\right) \leq A\left(\sum_{i=1}^{n} a_{i} f\left(x_{i}\right)\right)=\sum_{i=1}^{n} a_{i} f\left(x_{i}\right)$. This proves the last inequality of (3.1).

Remark 2. We note that Theorem 2.3 in [2] is the special case of Theorem 1 as $n=2, a_{1}=a_{2}=\frac{1}{2}, g(t)=t$ and $h(t)=t$.

Theorem 2. Under the conditions of Lemma 1 , if $x=\left\{x_{1}, x_{2}, \ldots, x_{n}\right\}$ is a sequence in $C$ such that $x_{i} \neq x_{i+1}, i=1,2, \ldots, n$, and $x_{n+1}=x_{1}$, then

$$
\begin{aligned}
f\left(\sum_{i=1}^{n} a_{i} x_{i}\right) & \leq \sum_{i=1}^{n} a_{i} f\left(\left[1-\frac{a}{2 a_{i}}\right] x_{i}+\frac{a}{2 a_{i}} x_{i+1}\right) \\
& \leq \sum_{i=1}^{n} \frac{a_{i}^{2}}{a\left(x_{i+1}-x_{i}\right)} \int_{x_{i}}^{x_{i}+\frac{a}{a_{i}}\left(x_{i+1}-x_{i}\right)} f(t) d t \\
& \leq \sum_{i=1}^{n} a_{i} f\left(x_{i}\right) .
\end{aligned}
$$

Proof. Let $A=\frac{1}{n a} \int_{0}^{n a}, E=[0, n a], g(t)=t$ and $h(t)=t$. Then

$$
\begin{aligned}
A\left(\Phi_{x}(h)\right) & =\frac{1}{n a} \int_{0}^{n a} \sum_{i=1}^{n} a_{i} f\left(\left[1-\frac{t}{n a_{i}}\right] x_{i}+\frac{t}{n a_{i}} x_{i+1}\right) d t \\
& =\sum_{i=1}^{n} \frac{a_{i}^{2}}{a\left(x_{i+1}-x_{i}\right)} \int_{x_{i}}^{x_{i}+\frac{a}{a_{i}}\left(x_{i+1}-x_{i}\right)} f(t) d t
\end{aligned}
$$

and

$$
\begin{aligned}
\Phi_{x}(A(h)) & =\sum_{i=1}^{n} a_{i} f\left(\left[1-\frac{\frac{1}{n a} \int_{0}^{n a} t d t}{n a_{i}}\right] x_{i}+\frac{\frac{1}{n a} \int_{0}^{n a} t d t}{n a_{i}} x_{i+1}\right) \\
& =\sum_{i=1}^{n} a_{i} f\left(\left[1-\frac{a}{2 a_{i}}\right] x_{i}+\frac{a}{2 a_{i}} x_{i+1}\right) .
\end{aligned}
$$

Using (3.1), we obtain

$$
f\left(\sum_{i=1}^{n} a_{i} x_{i}\right) \leq \sum_{i=1}^{n} a_{i} f\left(\left[1-\frac{a}{2 a_{i}}\right] x_{i}+\frac{a}{2 a_{i}} x_{i+1}\right)
$$




$$
\begin{aligned}
& \leq \sum_{i=1}^{n} \frac{a_{i}^{2}}{a\left(x_{i+1}-x_{i}\right)} \int_{x_{i}}^{x_{i}+\frac{a}{a_{i}}\left(x_{i+1}-x_{i}\right)} f(t) d t \\
& \leq \sum_{i=1}^{n} a_{i} f\left(x_{i}\right) .
\end{aligned}
$$

Remark 3. We note that the Hadamard's inequalities (1.1) is the special case of Theorem 2 when $n=2, x_{1}=a, x_{2}=b$, and $a_{1}=a_{2}=\frac{1}{2}$.

Tehorem 3. Under the conditions of Theorem 2, let $H:[0,1] \rightarrow R$ be a function defined by

$$
H(t)=\sum_{i=1}^{n} \frac{a_{i}^{2}}{a\left(x_{i+1}-x_{i}\right)} \int_{x_{i}}^{x_{i}+\frac{a}{a_{i}}\left(x_{i+1}-x_{i}\right)} f\left(t x+(1-t) \sum_{j=1}^{n} a_{j} x_{j}\right) d x .
$$

Then (1) $H$ is convex on $[0,1]$,

$$
\text { (2) } \begin{aligned}
f\left(\sum_{i=1}^{n} a_{i} x_{i}\right) & =H(0)=\min _{t \in[0,1]} H(t) \leq H(t) \\
& \leq \max _{t \in[0, i]} H(t)=H(1)=\sum_{i=1}^{n} \frac{a_{i}^{2}}{a\left(x_{i+1}-x_{i}\right)} \int_{x_{i}}^{x_{i}+\frac{a}{a_{i}}\left(x_{i+1}-x_{i}\right)} f(x) d x \\
& \leq \sum_{i=1}^{n} a_{i} f\left(x_{i}\right)
\end{aligned}
$$

for all $t \in[0,1]$,

(3) $H$ is increasing on $[0,1]$.

Proof. Let $t_{1}, t_{2} \in[0,1]$ and $\alpha, \beta \geq 0$ with $\alpha+\beta=1$. Since $f$ is convex on $C$, we have

$$
\begin{aligned}
& H\left(\alpha t_{1}+\beta t_{2}\right) \\
= & \sum_{i=1}^{n} \frac{a_{i}^{2}}{a\left(x_{i+1}-x_{i}\right)} \int_{x_{i}}^{x_{i}+\frac{a}{a_{i}}\left(x_{i+1}-x_{i}\right)} f\left(\left(\alpha t_{1}+\beta t_{2}\right) x+\left(1-\alpha t_{1}-\beta t_{2}\right) \sum_{j=1}^{n} a_{j} x_{j}\right) d x \\
= & \sum_{i=1}^{n} \frac{a_{i}^{2}}{a\left(x_{i+1}-x_{i}\right)} \\
& \times \int_{x_{i}}^{x_{i}+\frac{a}{a_{i}}\left(x_{i+1}-x_{i}\right)} f\left(\alpha\left[t_{1} x+\left(1-t_{1}\right) \sum_{j=1}^{n} a_{j} x_{j}\right]+\beta\left[t_{2} x+\left(1-t_{2}\right) \sum_{j=1}^{n} a_{j} x_{j}\right]\right) d x \\
\leq & \alpha \sum_{i=1}^{n} \frac{a_{i}^{2}}{a\left(x_{i+1}-x_{i}\right)} \int_{x_{i}}^{x_{i}+\frac{a}{a_{i}}\left(x_{i+1}-x_{i}\right)} f\left(t_{1} x+\left(1-t_{1}\right) \sum_{j=1}^{n} a_{j} x_{j}\right) d x
\end{aligned}
$$




$$
\begin{aligned}
& \quad+\beta \sum_{i=1}^{n} \frac{a_{i}^{2}}{a\left(x_{i+1}-x_{i}\right)} \int_{x_{i}}^{x_{i}+\frac{a}{a_{i}}\left(x_{i+1}-x_{i}\right)} f\left(t_{2} x+\left(1-t_{2}\right) \sum_{j=1}^{n} a_{j} x_{j}\right) d x \\
& =\alpha H\left(t_{1}\right)+\beta H\left(t_{2}\right) .
\end{aligned}
$$

This completes the proof of (1).

Now, observe that $H(0)=f\left(\sum_{i=1}^{n} a_{i} x_{i}\right)$ and $H(1)=\sum_{i=1}^{n} \frac{a_{i}^{2}}{a\left(x_{i}+1-x_{i}\right)}$ $\int_{x_{i}}^{x_{i}+\frac{a}{a_{i}}\left(x_{i+1}-x_{i}\right)} f(x) d x$. Using the convexity of $f$ and the inequality (3.2), we have

$$
\begin{aligned}
H(t)= & \sum_{i=1}^{n} \frac{a_{i}^{2}}{a\left(x_{i+1}-x_{i}\right)} \int_{x_{i}}^{x_{i}+\frac{a}{a_{i}}\left(x_{i+1}-x_{i}\right)} f\left(t x+(1-t) \sum_{j=1}^{n} a_{j} x_{j}\right) d x \\
\leq & t \sum_{i=1}^{n} \frac{a_{i}^{2}}{a\left(x_{i+1}-x_{i}\right)} \int_{x_{i}}^{x_{i}+\frac{a}{a_{i}}\left(x_{i+1}-x_{i}\right)} f(x) d x \\
& \quad+(1-t) \sum_{i=1}^{n} \frac{a_{i}^{2}}{a\left(x_{i+1}-x_{i}\right)} \int_{x_{i}}^{x_{i}+\frac{a}{a_{i}}\left(x_{i+1}-x_{i}\right)} f\left(\sum_{j=1}^{n} a_{j} x_{j}\right) d x \\
= & t \sum_{i=1}^{n} \frac{a_{i}^{2}}{a\left(x_{i+1}-x_{i}\right)} \int_{x_{i}}^{x_{i}+\frac{a}{a_{i}}\left(x_{i+1}-x_{i}\right)} f(x) d x+(1-t) f\left(\sum_{j=1}^{n} a_{j} x_{j}\right) \\
\leq & \sum_{i=1}^{n} \frac{a_{i}^{2}}{a\left(x_{i+1}-x_{i}\right)} \int_{x_{i}}^{x_{i}+\frac{a}{a_{i}}\left(x_{i+1}-x_{i}\right)} f(x) d x \leq \sum_{i=1}^{n} a_{i} f\left(x_{i}\right),
\end{aligned}
$$

for all $t \in[0,1]$.

On the other hand, let $y_{i}=t x_{i}+(1-t) \sum_{j=1}^{n} a_{j} x_{j}, 1 \leq i \leq n$, and $y_{n+1}=y_{1}$, then

$$
\begin{aligned}
H(t) & =\sum_{i=1}^{n} \frac{a_{i}^{2}}{a\left(y_{i+1}-y_{i}\right)} \int_{y_{i}}^{y_{i}+\frac{a}{a_{i}}\left(y_{i+1}-y_{i}\right)} f(y) d y \\
& \geq f\left(\sum_{j=1}^{n} a_{i} y_{i}\right)=f\left(\sum_{i=1}^{n} a_{i} x_{i}\right),
\end{aligned}
$$

for all $t \in[0,1]$.

This completes the proof of (2).

Finally, let $0<t<u \leq 1$. Since $H$ is convex on $[0,1]$ and $H(t) \geq H(0)$, we have

$$
\frac{H(u)-H(t)}{u-t} \geq \frac{H(t)-H(0)}{t} \geq 0,
$$

that is $H(t) \leq H(u)$.

This completes the proof of (3).

Remark 4. We note that Theorem 1 in [3] is the special case of Theorem 3 when $n=2, a_{1}=a_{2}=\frac{1}{2}$. 
Theorem 4. Under the conditions of Theorem 2 , let $K:[0,1] \rightarrow R$ be a function defined by

$$
\begin{gathered}
K(t)=\sum_{i=1}^{n} \frac{a_{i}^{2}}{2 a\left(x_{i+1}-x_{i}\right)} \int_{x_{i}}^{x_{i}+\frac{a}{a_{i}}\left(x_{i+1}-x_{i}\right)}\left\{f\left(\left[\frac{1-t}{2}\right] x+\left[\frac{1+t}{2}\right] x_{i}\right)\right. \\
\left.\quad+f\left(\left[\frac{1-t}{2}\right] x+\left[\frac{1+t}{2}\right]\left[x_{i}+\frac{a}{a_{i}}\left(x_{i+1}-x_{i}\right)\right]\right)\right\} d x
\end{gathered}
$$

Then $K$ is convex, increasing on $[0,1]$ and

$$
\sum_{i=1}^{n} \frac{a_{i}^{2}}{a\left(x_{i+1}-x_{i}\right)} \int_{x_{i}}^{x_{i}+\frac{a}{a_{i}}\left(x_{i+1}-x_{i}\right)} f(x) d x=K(0) \leq K(t) \leq K(1) \leq \sum_{i=1}^{n} a_{i} f\left(x_{i}\right)
$$

for all $t \in[0,1]$.

Proof. Using Lemma 2 , it is easy to see that $K$ is convex and increasing on $[0,1]$. Now,

$$
\begin{aligned}
K(0)= & \sum_{i=1}^{n} \frac{a_{i}^{2}}{a\left(x_{i+1}-x_{i}\right)} \int_{x_{i}}^{x_{i}+\frac{a}{a_{i}}\left(x_{i+1}-x_{i}\right)} f(x) d x \\
K(1)= & \sum_{i=1}^{n} \frac{a_{i}^{2}}{a\left(x_{i+1}-x_{i}\right)} \\
& \int_{x_{i}}^{x_{i}+\frac{a}{a_{i}}\left(x_{i+1}-x_{i}\right)} \frac{1}{2}\left[f\left(x_{i}\right)+f\left(x_{i}+\frac{a}{a_{i}}\left(x_{i+1}-x_{i}\right)\right)\right] d x \\
= & \sum_{i=1}^{n} \frac{a_{i}\left[f\left(x_{i}\right)+f\left(x_{i}+\frac{a}{a_{i}}\left(x_{i+1}-x_{i}\right)\right)\right]}{2} \\
= & \frac{1}{2} \sum_{i=1}^{n} a_{i} f\left(x_{i}\right)+\frac{1}{2} \sum_{i=1}^{n} a_{i} f\left(x_{i}+\frac{a}{a_{i}}\left(x_{i+1}-x_{i}\right)\right) \\
\leq & \sum_{i=1}^{n} a_{i} f\left(x_{i}\right) .
\end{aligned}
$$

This completes the proof.

Remark 5. Let $a_{i}=\frac{1}{n}(i=1,2, \ldots, n)$ and $x_{n+1}=x_{1}<x_{2}<\cdots<x_{n}$. Then, from Theorem 3 and Theorem 4, we have

$$
H(t)=\sum_{i=1}^{n} \frac{1}{n\left(x_{i+1}-x_{i}\right)} \int_{x_{i}}^{x_{i+1}} f\left(t x+\frac{1-t}{n} \sum_{j=1}^{n} x_{j}\right) d x
$$

and 


$$
\begin{aligned}
K(t)=\sum_{i=1}^{n} & \frac{1}{2 n\left(x_{i+1}-x_{i}\right)} \int_{x_{i}}^{x_{i+1}}\left\{f\left(\left[\frac{1-t}{2}\right] x+\left[\frac{1+t}{2}\right] x_{i}\right)\right. \\
& \left.+f\left(\left[\frac{1-t}{2}\right] x+\left[\frac{1+t}{2}\right] x_{i+1}\right)\right\} d x
\end{aligned}
$$

such that $H$ and $K$ are convex increasing on $[0,1]$ and

$$
\begin{aligned}
f\left(\frac{1}{n} \sum_{i=1}^{n} x_{i}\right) & =H(0) \leq H(t) \\
\leq H(1) & =\sum_{i=1}^{n} \frac{1}{n\left(x_{i+1}-x_{i}\right)} \int_{x_{i}}^{x_{i+1}} f(x) d x=K(0) \\
& \leq K(t) \leq K(1)=\frac{1}{n} \sum_{i=1}^{n} f\left(x_{i}\right) .
\end{aligned}
$$

\section{References}

[1] P. R. Beesack and J. E. Pecaric, "On Jessen's inequality for convex functions," J. Math. Anal. Appl., 110 (1995), 536-552.

[2] S. S. Dragomir, "A refinement of Hadamard's inequality," Tamkang Journal of Math., 4(1)(1993).

[3] S. S. Dragomir, "Two mappings in connection to Hadamard's inequalities," J. Math. Anal. Appl., 167 (1992), 49-56.

[4] G. S. Yang and M. C. Hong. "A note on Hadamard's inequality," (To appear, Tamkang J. Math.).

Department of Mathematics, Tamkang University, Tamsui, Taiwan.

Department of Mathematics, Tamsui Oxford University College, Tamsui, Taiwan. 